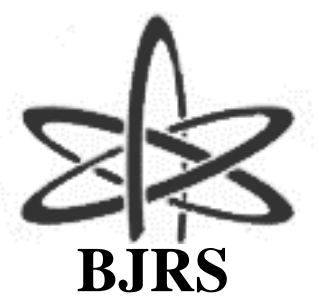

\author{
BRAZILIAN JOURNAL \\ $\mathrm{OF}$ \\ RADIATION SCIENCES \\ 06-02-B (2018) 01-16
}

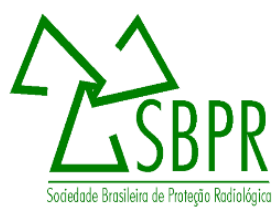

\title{
Panorama da segurança física de fontes radioativas no Brasil
}

\author{
Lima $^{\text {a,b }}$ A. R., Tavares ${ }^{a}$ R. L. A, Filho ${ }^{a}$ J. S. M., Da Silva ${ }^{b}$ F. C. A. \\ ${ }^{a}$ Comissão Nacional de Energia Nuclear, 22.290-901, Rio de Janeiro - RJ, Brasil \\ ${ }^{b}$ Instituto de Radioproteção e Dosimetria/CNEN, 22.783-127, Rio de Janeiro - RJ, Brasil \\ alexandre.lima@cnen.gov.br
}

\section{RESUMO}

A ameaça de "terrorismo radiológico" foi reconhecida mundialmente após o evento de 11 de setembro de 2001. Fontes radioativas podem ser utilizadas para construção de DDR - Dispositivos de Dispersão Radiológica ("bomba suja"). Estudos demonstram que o uso de um DDR poderia causar danos à saúde, psicossociais e prejuízos econômicos e ambientais. O Brasil acompanha essa preocupação mundial, pois possui amplo parque médicoindustrial que utiliza fontes radioativas. Este trabalho apresenta um panorama sobre a segurança física de fontes radioativas no país, baseado no inventário de instalações radiativas, comparando os requisitos regulatórios nacionais às recomendações internacionais. No Brasil existem aproximadamente 2.500 instalações radiativas, com cerca de 500 fontes radioativas Categoria 1 e 2, que são a maior preocupação em termos de segurança física. A norma brasileira de licenciamento aborda somente alguns aspectos de proteção física, não apresentando orientação clara para elaboração e implantação de sistemas de proteção física, não atendendo às recomendações internacionais. Para o Brasil se inserir no cenário mundial de segurança física de fontes radioativas torna-se premente a elaboração de legislação específica, com critérios regulatórios bem definidos. A falta de requisitos mais detalhados dificulta avaliação regulatória mais criteriosa sobre as condições de proteção física das instalações, seja por meio da avaliação de planos e demais documentos de proteção física ou pela realização de inspeções regulatórias.

Palavras-chave: segurança física, terrorismo radiológico, bomba suja. 


\begin{abstract}
The threat of "radiological terrorism" has been widely recognized after September $11^{\text {th }}, 2001$ event. Radioactive $^{2}$ sources can be used for construction of RDD - Radiological Dispersion Devices. Studies show that the use of a RDD could cause health, psychosocial, economic and environmental damages. Brazil follows this worldwide concern, since it has a large number of facilities that use radioactive sources. There are in the country around 2,500 radiological facilities in operation, with approximately 500 Category 1 and 2 radioactive sources, which are the most important concern for security. This work presents an overview of the security of radioactive sources in Brazil based on the inventory of radiological facilities, comparing national regulatory requirements to international recommendations. Currently Brazilian regulatory standards address only some aspects of physical protection, neither providing clear orientation for elaboration and implementation of a physical protection system, not completely addressing the most recent international recommendations. This work demonstrates the urgent need of improvement of the security of radioactive sources, through a specific regulation with well-defined criteria. The lack of more detailed requirements hampers a more adequate regulatory assessment of the physical protection conditions of the facilities, either through the evaluation of physical protection plans and other documents or by regulatory inspections.
\end{abstract}

Keywords: radiological security, radiological terrorism, dirty bomb.

\title{
1. INTRODUÇÃ̃
}

A preocupação crescente da comunidade internacional em relação à segurança física de fontes radioativas resultou no fortalecimento, a nível mundial, da aplicação de requisitos de proteção e recursos associados através da adoção de novos parâmetros e metodologias para a concepção e implantação de novas medidas e sistemas [1].

A ameaça de terrorismo radiológico é reconhecida mundialmente, pois terroristas vêm de fato tentando obter fontes radioativas necessárias ao desenvolvimento de um DDR - Dispositivos de Dispersão Radiológicos, comumente conhecidos como "bomba suja". Estima-se que tais artefatos tenham potencial de causar prejuízos sociais, econômicos e ambientais consideráveis, além de danos psicológicos à saúde dos afetados. O Brasil acompanha essa preocupação mundial, pois possui amplo parque industrial e médico que faz uso de fontes radioativas de alta atividade [1]. 
Após o atentado de 11/9 no World Trade Center (EUA), a segurança física de fontes radioativas vem se tornando um assunto amplamente discutido internacionalmente, através de organizações como a AIEA - Agência Internacional de Energia Atômica, organismo das Nações Unidas, que vem trabalhando para auxiliar os Estados Membros a introduzir medidas de segurança física de forma consistente e abrangente. Entretanto, apenas uma minoria dos Estados implantou ações e medidas realmente eficazes de segurança física das fontes radioativas, em especial aqueles que se sentem mais ameaçados por ações terroristas fazendo uso de "bombas sujas", o que faz que estes se autodenominem "Estados-alvo" [2].

Através dos esforços da AIEA e de alguns programas nacionais e internacionais, registrou-se progresso relevante na obtenção da segurança física das fontes radioativas nos últimos dez anos. Contudo, tais esforços são comumente impactados por vários desafios internos dos próprios Estados, tais como infraestrutura, arcabouço legal e recursos em geral [2].

A AIEA possui grupos de trabalho, com participação dos Estados Membros, que tratam de assuntos relacionados à segurança física de fontes radioativas. Inclui discussão e troca de informações de forma a fortalecer e disseminar a cultura de segurança física, permitindo o fortalecimento das infraestruturas nacionais que exerçam o controle regulatório das fontes [3].

Ademais, a AIEA elabora e disponibiliza aos Estados Membros documentos internacionais, como guias e recomendações, que apresentam conceitos, parâmetros e diretrizes atualizadas para elaboração de requisitos normativos, ficando a cargo de cada Estado adotar ou não tais recomendações $[4,5]$.

O presente estudo apresenta um panorama da segurança física de fontes radioativas no Brasil, baseado no inventário de instalações radiativas, comparando os requisitos regulatórios nacionais em vigor com as recomendações internacionais.

\section{MATERIAIS E MÉTODOS}

Para a elaboração do estudo, foram utilizadas a base de dados de fontes radioativas do órgão regulador [6], o status normativo atual e as recomendações internacionais. 


\subsection{Recomendações internacionais}

Os termos Safety e Security não possuem tradução em português que os diferencie de fato, pois ambos significam "segurança”. Com isso, nas áreas radiológica e nuclear, traduz-se o termo Safety como segurança radiológica e o Security como segurança física. Em resumo, a segurança radiológica protege os indivíduos dos efeitos nocivos da radiação ionizante e a segurança física protege a fonte radioativa do indivíduo mal-intencionado [7].

O Código de Conduta [7], publicado em 2004, foi o primeiro documento internacional a abordar a segurança física e radiológica de fontes radioativas O Código aplica-se a todas as fontes radioativas "perigosas", ou seja, àquelas que possam representar maior risco radiológico para indivíduos, sociedade e meio-ambiente. Importante ressaltar que o Código não é mandatório para os EstadosMembros, mas demonstra consenso internacional sobre a necessidade de um forte programa regulatório que inclua aplicação de requisitos normativos para a segurança física de fontes radioativas [7].

O Estado, através dos órgãos ligados à segurança (ver Figura 1), deve definir sua ameaça interna. Esse processo inicia-se com uma estimativa de possíveis ameaças, que é uma análise que documenta - a nível nacional - as motivações, intenções e capacidades de potenciais adversários críveis que possam causar danos através da sabotagem de uma instalação radiativa, ou a remoção não autorizada de uma fonte radioativa para fins maléficos [4].

Figura 1: Órgãos Envolvidos na Estimativa de Ameaça.

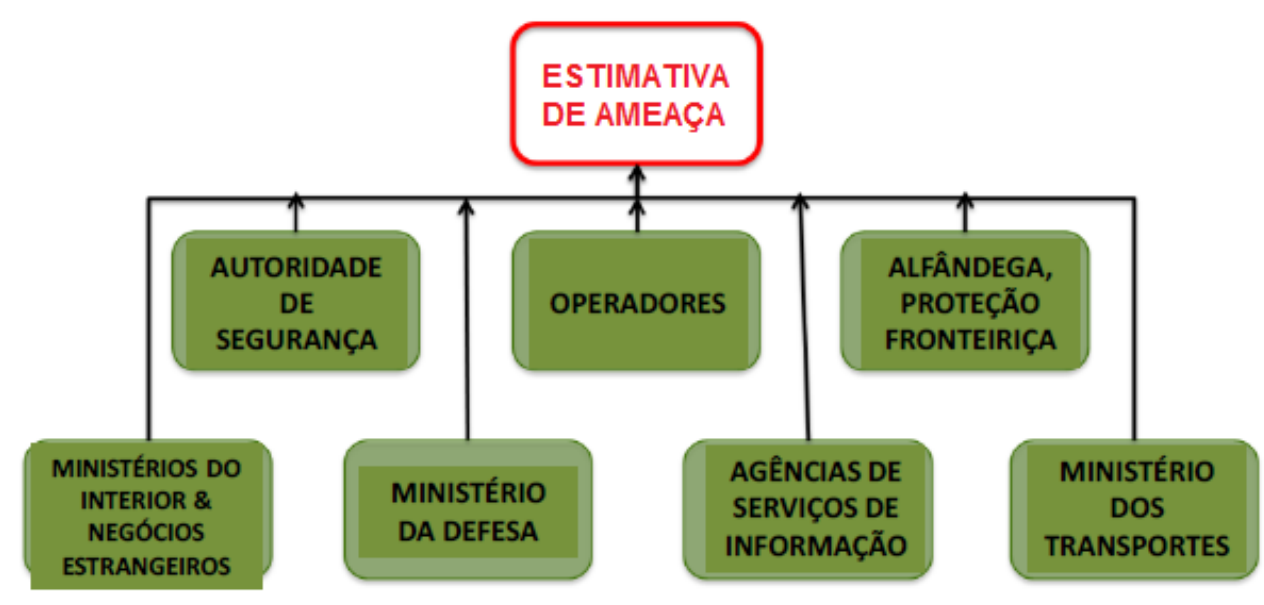


Ademais, o Estado deve adotar medidas adequadas para garantir que as fontes radioativas em seu território, ou sob sua jurisdição ou controle, sejam protegidas de forma segura durante suas vidas úteis. Isso inclui a promoção de uma cultura de segurança física em relação às fontes radioativas, educação e capacitação aos reguladores e licenciados e, principalmente, a manutenção de uma infraestrutura legislativa e regulatória eficaz [4,5].

O Estado deve desenvolver, implantar e manter atuante um Regime de Segurança Nuclear (ver Figura 2), sendo capaz de oferecer, a nível nacional, proteção às pessoas, à sociedade e ao meio ambiente de riscos e danos que possam ser causados caso ocorra algum evento relacionado à segurança física das fontes radioativas [5].

Figura 2: Estrutura da Segurança Física do Estado.

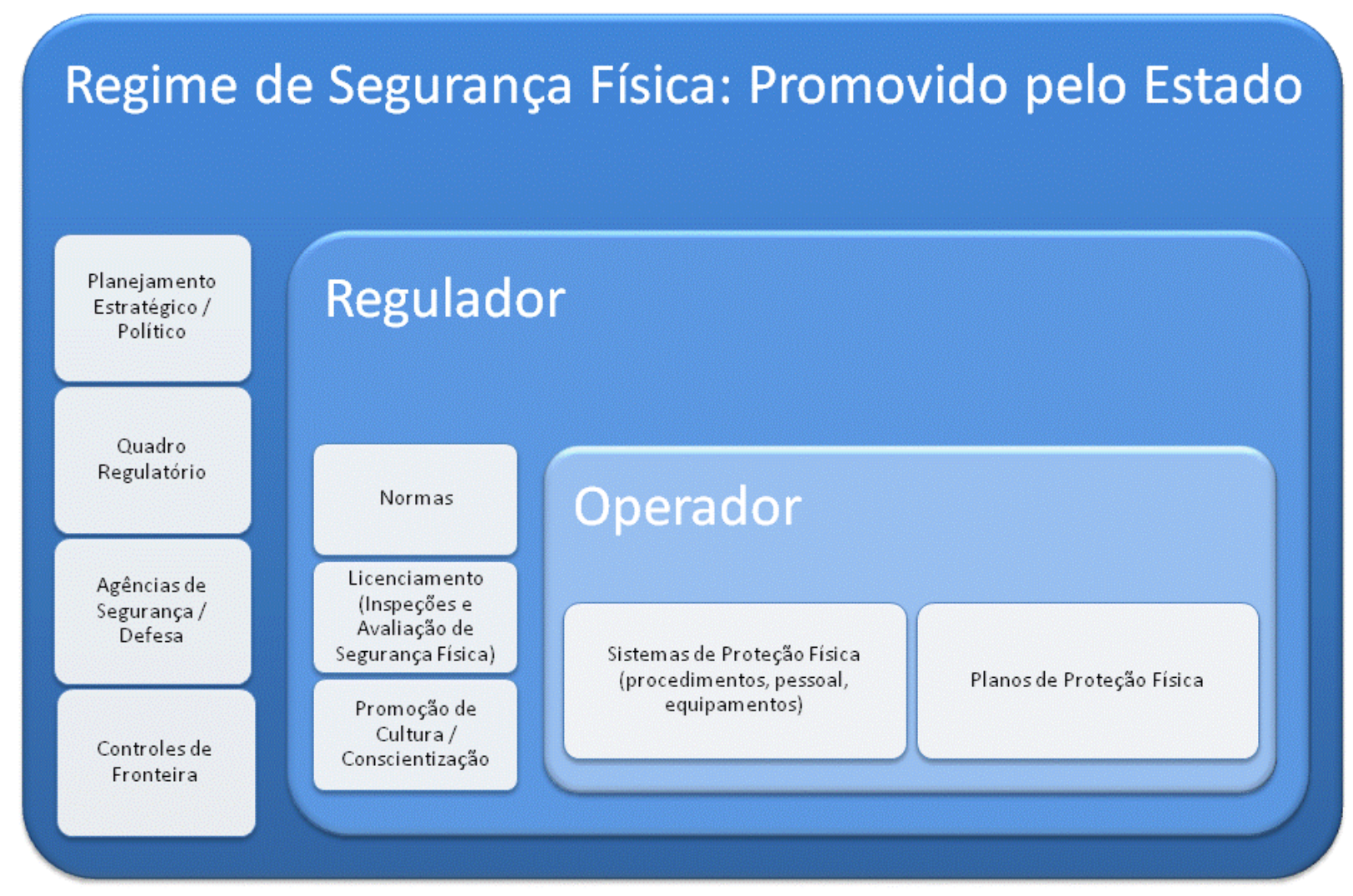


Neste regime, as responsabilidades da autoridade reguladora na área nuclear e radiológica devem ser claramente definidas e atribuídas, assim como, de outras autoridades competentes do governo, incluindo agências de inteligência, ministérios de defesa, transporte e de relações exteriores, polícias, aduanas, e outras autoridades com responsabilidades relacionadas à segurança nacional, prevendo adequada integração, coordenação e comunicação [5].

Compete ao Estado designar um órgão regulador na área nuclear e radiológica, este responsável pelo licenciamento e fiscalização das instalações radiativas, que tenha um arcabouço legal e independência claramente definida em relação ao licenciado [5].

O termo "proteção física" é utilizado quando se trata exclusivamente de medidas adotadas pelo licenciado no âmbito da instalação, visando proteger fisicamente suas fontes radioativas. As medidas de "segurança física" são aplicadas em âmbito governamental, através dos seus órgãos ligados à segurança ou pelo regime de segurança física do Estado [8].

Os licenciados devem ser responsáveis pela implantação e manutenção de medidas de proteção física para fontes radioativas de acordo com os requisitos normativos estabelecidos pelo regulador. Ademais, os licenciados devem incluir a confiabilidade nos indivíduos envolvidos no controle e operação de fontes, através de verificação de antecedentes, antes mesmo que o indivíduo tenha acesso autorizado a locais onde estas são usadas ou armazenadas ou qualquer informação sensível relacionada às mesmas [4].

A complexidade e dinamismo das ameaças a instalações críticas, tanto em termos globais quanto locais, ensejam atualizações e adaptações aos requisitos normativos referentes à proteção física, de forma a manter a eficácia dos sistemas de proteção das instalações. Esses devem estar em consonância com o disposto na Resolução 1540 do Conselho de Segurança da ONU - Organização das Nações Unidas, tornada juridicamente vinculante (obrigatória) para todos os Estados-Membros das Nações Unidas, sendo no Brasil consolidada pelo Decreto 7722, de 20 de abril de 2012 [9].

\subsection{Concepção do sistema de proteção física}

De forma a garantir a proteção física das fontes radioativas, o licenciado deve prover um sistema de proteção física eficaz e eficiente com recursos humanos, técnicos e tecnológicos adequados, ou 
seja, com pessoas, procedimentos e equipamentos integrados capazes de prevenir e responder a atos maléficos (roubo, sabotagem, acesso não autorizado, transferência ilegal etc.) [7].

O sistema deve executar as funções básicas da proteção física (ver Tabela 1) com gerenciamento seguro, implantação de sistemas que impossibilitem ou retardem o acesso não autorizado, a detecção caso tal acesso não autorizado ocorra, e a garantia de medidas para mitigar as consequências e recuperar as fontes radioativas [4].

Tabela 1: Funções Básicas de Proteção Física [4].

\begin{tabular}{|c|c|}
\hline Função & Definição \\
\hline Dissuasão & $\begin{array}{l}\text { É a percepção pelo adversário de que será muito difícil o acesso não } \\
\text { autorizado até a fonte radioativa, e as consequências que causariam tal ato } \\
\text { não justificam o risco da tentativa de tal acesso. Exemplos de elementos } \\
\text { dissuasórios incluem a presença de guardas armados visíveis, câmeras, avisos } \\
\text { e barreiras físicas. }\end{array}$ \\
\hline Detecção & $\begin{array}{l}\text { É a descoberta de uma ação de um adversário interno ou externo, visando às } \\
\text { fontes a proteger, seja tal ação realizada de forma ostensiva ou oculta. Tal } \\
\text { descoberta pode se dar através de sensores, observação humana ou outras } \\
\text { formas equivalentes. A função inclui a certificação, ou seja, confirmação da } \\
\text { natureza do ato. }\end{array}$ \\
\hline Retardo & $\begin{array}{l}\text { Consiste em atrasar o ato maléfico por tempo suficiente para a ação adequada } \\
\text { da força de resposta. Pode se dar através de barreiras físicas, cercas, grades } \\
\text { ou outros meios físicos. }\end{array}$ \\
\hline Resposta & $\begin{array}{l}\text { Contempla as ações adotadas para interromper a ato maléfico. Tal } \\
\text { interrupção pode se dar, por exemplo, por meio de pessoal em número } \\
\text { suficiente que chegue a tempo e no local correto para impedir o ato. }\end{array}$ \\
\hline $\begin{array}{c}\text { Gestão de } \\
\text { Segurança Física }\end{array}$ & $\begin{array}{l}\text { Consiste em obter recursos para a manutenção do sistema de proteção física } \\
\text { da instalação radiativa, além de garantir a implantação e cumprimento de } \\
\text { procedimentos, políticas, registros e planos para a proteção física das fontes } \\
\text { radioativas. }\end{array}$ \\
\hline
\end{tabular}


Complementando a Tabela 1, a AIEA [4] recomenda seis tópicos de gestão de segurança física:

- Controle de acesso, para limitar o acesso às fontes radioativas;

- Confiabilidade, para verificar os antecedentes dos indivíduos que terão acesso às fontes radioativas;

- Proteção da informação sensível, para evitar o acesso não autorizado;

- Plano de contingência, documento que descreve as medidas de segurança que devem ser adotadas em caso de eventos relacionados ao roubo da fonte radioativa ou sabotagem da instalação;

- Notificação dos eventos, para definir e especificar o que deve ser reportado às autoridades reguladoras e outros órgãos, caso ocorra um evento de segurança física, incluindo responsáveis e prazos; e

- Plano de proteção física, considerado as seguintes premissas de projeto:

Descrever os elementos e subsistemas de um sistema de proteção física instalados, incluindo sua manutenção e condições de operacionalidade.

$\checkmark$ Prover defesa em profundidade, ou seja, implantar sucessivas camadas de barreiras que um adversário deve ter que ultrapassar, em grau crescente de dificuldade, para a consecução do ato maléfico.

$\checkmark$ Demonstrar uma proteção física balanceada, independente da rota ou modo de execução do ato maléfico, havendo um grau eficaz de proteção em volta da fonte radioativa.

$\checkmark$ Detectar em tempo hábil o roubo ou perda da fonte radioativa de forma a responder adequadamente;

$\checkmark$ Prover uma resposta eficaz e gradual à ameaça sobre as fontes radioativas.

O conceito de "abordagem gradual" é amplamente utilizado na literatura [2,4,5] como importante consideração para a segurança física. Os requisitos normativos devem ser nele baseados, levando-se em conta os princípios de gestão de risco, incluindo considerações tais como a estimativa de ameaças (fornecida pelo Estado) e a atratividade relativa da fonte radioativa para um ato maléfico. Fatores como quantidade, atividade, propriedade física e química, mobilidade, disponibilidade e acessibilidade à fonte radioativa na instalação devem ser considerados [5]. 
Baseado na gestão de risco e na abordagem gradual recomenda-se ao regulador a criação de um enquadramento da instalação em três níveis de proteção física distintos, denominados "A", "B" e "C". O nível de proteção física "A" corresponde ao grau de maior exigência, sendo os demais níveis progressivamente menos exigentes [4].

Cada nível tem seu objetivo específico, definindo as características de projeto, implantação e operação do sistema de proteção física. Para atingir tais objetivos é necessário adotar as medidas de proteção previstas no nível correspondente, através das funções básicas relacionadas na Tabela 1 [4].

Para determinação do nível utiliza-se o sistema de categorização de fontes radioativas descrito no Guia de Segurança RS-G-1.9 [10], que fornece uma base consistente e sistemática para identificação e classificação das mesmas de acordo com seu nível de periculosidade. Tal sistema baseia-se nos efeitos para a saúde que resultam da exposição não controlada a uma fonte radioativa, podendo ser atribuído categorias de 1 a 5, sendo a categoria 1 a mais perigosa [10].

De forma prática, a categorização de uma fonte radioativa pode ser obtida pela relação (ou divisão) entre o valor da atividade da fonte "A" e o valor "D" (Dangerous). O valor "D" é normalizado e específico para cada fonte radioativa, levando em consideração o risco associado a um efeito deletério após exposição. Quando a instalação radiativa possui fontes radioativas distintas, somam-se os resultados dos quocientes A/D relacionados a cada fonte [10].

Alternativamente, a categorização pode ser obtida por prática, sem levar em consideração a relação A/D. Com isso, as práticas são classificadas de acordo com o seu nível de periculosidade, conforme Tabela $2[4,10]$. 
Tabela 2: Níveis de Proteção Física Recomendadas para Fontes Radioativas (adaptado de [4,10]).

\begin{tabular}{ccc}
\hline Categoria & Arática/Fontes & Nível de \\
Proteção
\end{tabular}

Após a definição da categorização, para as categorias 1, 2 ou 3, aplica-se o nível de proteção física correspondente, A, B ou C, respectivamente. Às fontes Categoria 4 e 5 não são atribuídos um nível de proteção física, pois se presume que estas estejam adequadamente protegidas através da adoção de medidas de proteção radiológica [4].

Os níveis de proteção física da instalação podem ser reclassificados com base nas estimativas de ameaça ou dependendo da atratividade das fontes radioativas, a critério do regulador [4]. 


\section{RESULTADOS E DISCUSSÃO}

No Brasil existem aproximadamente 2.500 instalações radiativas, com cerca de 500 fontes radioativas categoria 1 e 2, que são a maior preocupação em termos de proteção física. As práticas enquadradas nessas categorias são, entre outras, irradiadores de grande porte e autoblindados, teleterapia e radiografia industrial.

Conforme registros obtidos no SIR - Sistema de Instalações Radiativas [6], acessado em 07/06/2018, o total de instalações médicas e industriais representa 73\% das instalações em operação. A distribuição de instalações radiativas em operação, por área de aplicação, é mostrada na Figura 3.

Figura 3: Instalações Radiativas Distribuídas por Área de Aplicação.

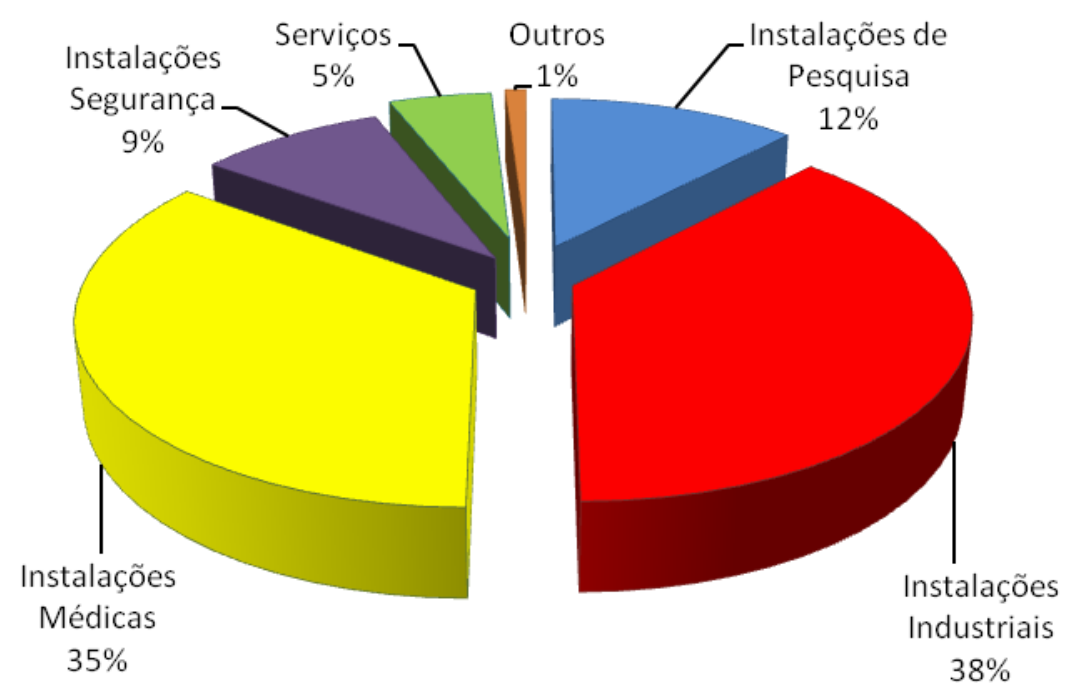

(Fonte: SIR/CNEN)

Atualmente a CNEN - Comissão Nacional de Energia Nuclear possui uma Norma que trata dos aspectos gerais do licenciamento de instalações radiativas, denominada CNEN NN 6.02 [11], que aborda os requisitos de proteção física. Esta Norma exige que o licenciado apresente um plano 
preliminar de proteção física para obtenção de uma licença de construção, e um plano de proteção física para a obtenção de uma autorização para operação da instalação radiativa.

Existem também duas normas específicas de proteção radiológica para instalações radiativas de radiografia industrial e radioterapia, que abordam que o licenciado deve adotar medidas relativas à proteção física das fontes radioativas, sendo as Normas CNEN NN 6.04 [12] e CNEN NN 6.10 [13], respectivamente.

A Seção VIII da Norma CNEN NN 6.04 estabelece para instalações radiativas de radiografia industrial que o licenciado deve elaborar um Programa de Proteção Física, em conformidade com a Norma CNEN NE 2.01 [14]. Entretanto, nesta Norma, os requisitos específicos de proteção física são derivados de regulamentos aplicados às instalações nucleares, dificultando sua aplicação direta em instalações que fazem uso de fontes radioativas.

A Norma CNEN NN 6.10, no Inciso X do seu Artigo 48, estabelece que as fontes radioativas de radioterapia fora de uso devem ser armazenadas em condições que garantam sua proteção física, no entanto não aborda mais detalhes sobre tais condições.

A Tabela 3 abaixo sintetiza e compara as recomendações internacionais com os requisitos normativos nacionais em vigor conforme os temas mais importantes de Proteção Física.

Tabela 3: Principais temas relacionados à segurança física de fontes radioativas.

\begin{tabular}{|c|c|c|c|}
\hline Tema & $\begin{array}{l}\text { Recomendações } \\
\text { Internacionais }\end{array}$ & $\begin{array}{c}\text { Requisitos Normativos } \\
\text { Nacionais }\end{array}$ & Comentários \\
\hline \multirow[t]{2}{*}{$\begin{array}{c}\text { Estimativa de } \\
\text { Ameaças }\end{array}$} & $\begin{array}{l}\text { O Estado deve fornecer uma } \\
\text { estimativa de ameaça com as } \\
\text { motivações, intenções e } \\
\text { capacidades de potenciais }\end{array}$ & $\begin{array}{l}\text { O Estado não fornece uma } \\
\text { estimativa qualitativa de } \\
\text { ameaças }\end{array}$ & $\begin{array}{c}\text { As medidas de } \\
\text { proteção física devem } \\
\text { ser adequadas à } \\
\text { ameaça identificada }\end{array}$ \\
\hline & adversários & Não há definição de ameaça & pela estimativa \\
\hline $\begin{array}{l}\text { Ameaças } \\
\text { Internas }\end{array}$ & $\begin{array}{l}\text { O regulador deve assegurar a } \\
\text { confiabilidade de indivíduos } \\
\text { com acesso autorizado a } \\
\text { fontes radioativas ou } \\
\text { informações sensíveis de }\end{array}$ & $\begin{array}{l}\text { Não há referência a este tipo } \\
\text { de ameaça }\end{array}$ & $\begin{array}{c}\text { Um indivíduo com } \\
\text { acesso autorizado a } \\
\text { instalações radiativas } \\
\text { ou a informações } \\
\text { sensíveis pode }\end{array}$ \\
\hline
\end{tabular}


segurança física, sendo uma

medida chave na mitigação

de possíveis ameaças cometer ou facilitar a

execução de um ato

maléfico

internas

\section{Conceitos:}

Cultura de

Segurança

Física,

Abordagem

Gradual, Defesa

em

Profundidade,

Defesa

Balanceada

\section{Funções de}

proteção física:

dissuasão,

detecção, Conforme preconizados nas

retardo, recomendações da IAEA

resposta e

gestão de

segurança física
Conforme preconizados nos documentos da AIEA
Não há os referidos conceitos
Os conceitos de segurança física devem ser adotados para garantir um sistema de proteção

física adequado e eficaz

$\begin{array}{cccc} & \text { Definido pela AIEA como } & \text { Dependendo do tipo de } & \text { O plano de proteção } \\ & \text { um documento - elaborado } & \text { instalação radiativa, a } & \text { física deve estar em } \\ \text { Plano de } & \text { pelo operador e avaliado } & \text { CNEN exige ao licenciado } & \text { conformidade com os } \\ \text { Proteção Física } & \text { pelo regulador - que } & \text { que apresente um plano de } & \text { requisitos normativos } \\ & \text { apresenta descrição } & \text { proteção física, mas não } & \text { e fornecer resposta ao } \\ & \text { detalhada dos aspectos de } & \text { estabelece requisitos } & \text { aumento dos níveis de } \\ & \text { proteção física da instalação } & \text { mínimos para sua } & \text { ameaça }\end{array}$




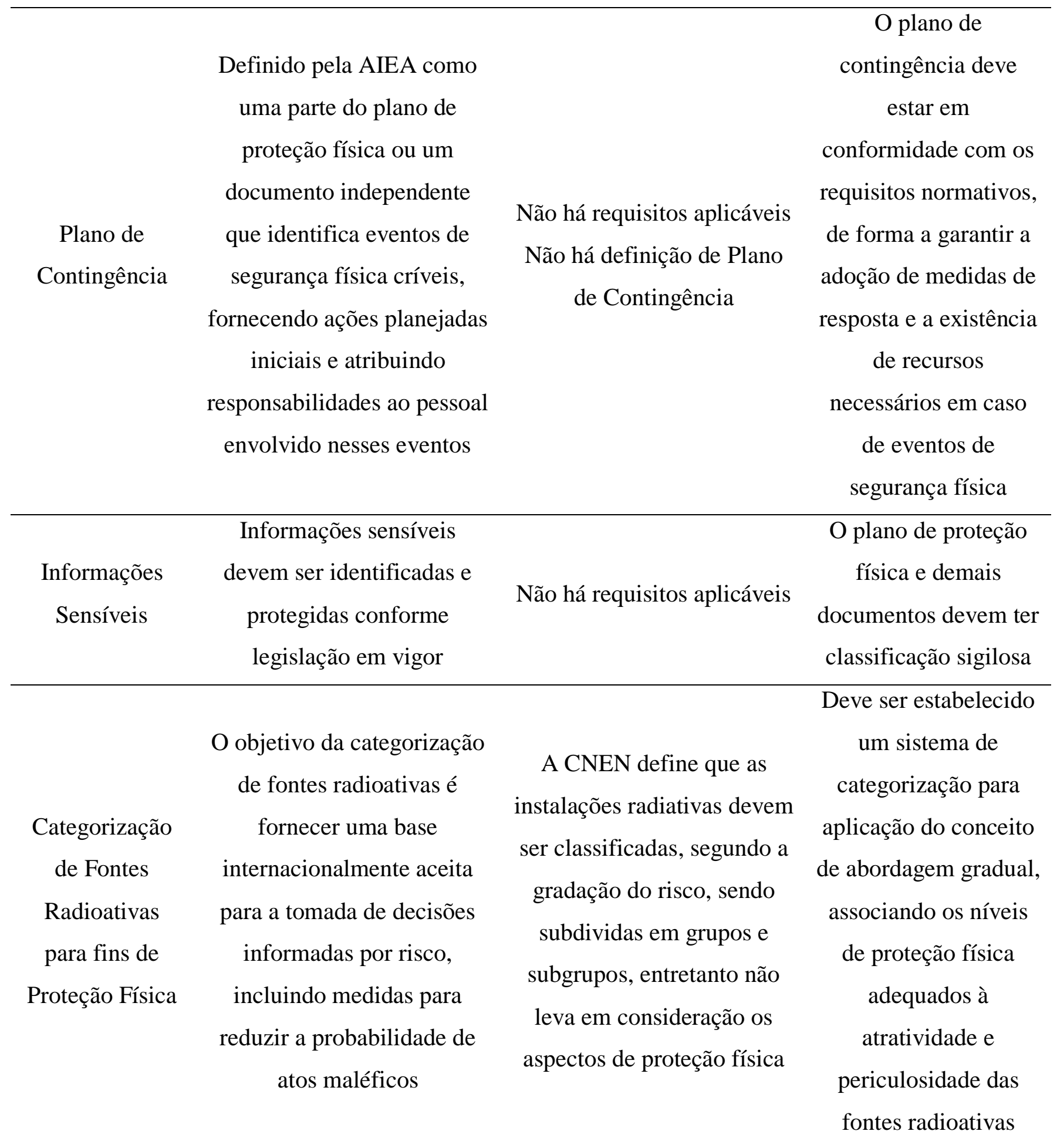




\section{CONCLUSÕES}

As normas brasileiras de licenciamento abordam aspectos de proteção física, entretanto não apresentam orientação clara para sua elaboração e implantação, não atendendo às recomendações internacionais.

A falta de requisitos mais detalhados dificulta uma avaliação regulatória mais criteriosa sobre as condições de proteção física das instalações, seja por meio da avaliação de planos e demais documentos de proteção física, ou por meio de inspeções regulatórias. Para o Brasil se inserir no cenário mundial de segurança física de fontes radioativas, torna-se premente a elaboração de uma legislação ou normatização específica. Essa deve conter requisitos normativos bem definidos, preferencialmente dispostos em um único documento, de forma ordenada e de acordo com as categorias das fontes radioativas e/ou os tipos de instalação, permitindo ao licenciado localizar e identificar seu enquadramento e, com isto, adotar as medidas adequadas de proteção física.

O presente estudo comparativo serviu de base técnica para tomada de decisão por parte da CNEN sobre o projeto de uma Norma específica de proteção física de fontes radioativas. A Norma encontrase em processo de elaboração através de uma Comissão de Estudos, com previsão de entrada em vigor em 2018. Sugere-se para trabalhos futuros a identificação e avaliação dos impactos desta nova Norma para o País, sob os pontos de vista do regulador e do operador.

\section{REFERÊNCIAS}

[1] LIMA A. R.; FILHO J. S. M.; MELLO L. A. New Brazilian Regulation for Security of Radioactive Sources, In: International Conference on Nuclear Security: Commitments and Actions, IAEA, Vienna, 2016.

[2] ENGLEFIELD, CHRIS. Radioactive Source Security: Why Do We Not Yet Have a Global Protection System? Nuclear Engineering and Technology. V. 46, p. 461-466, 2014. Disponível em: <http://www.sciencedirect.com/science/article/pii/S1738573315301133>. Acessado em: 04/04/2017. 
[3] IAEA - International Atomic Energy Agency. Division of Nuclear Security Homepage. Disponível em: <https://www.iaea.org/about/organizational-structure/department-of-nuclearsafety-and-security/division-of-nuclear-security>. Acessado em: 05/06/2018.

[4] IAEA - International Atomic Energy Agency. Implementing Guide on Security of Radioactive Sources. Nuclear Security Series No. 11, IAEA, Vienna, 2009.

[5] IAEA - International Atomic Energy Agency. Recommendations on Security of Radioactive Material and Associated Facilities. Nuclear Security Series No. 14, IAEA, Vienna, 2011.

[6] CNEN - Comissão Nacional de Energia Nuclear. Sistema de Instalações Radiativas da CNEN - SIR. CNEN, Brasil. Acessado em: 07/06/2018.

[7] IAEA - International Atomic Energy Agency. Code of Conduct Code of Conduct on the Safety and Security of Radioactive Sources, IAEA, Vienna, 2004

[8] IAEA - International Atomic Energy Agency. Physical Protection of Nuclear Material and Nuclear Facilities (Implementation of INFCIRC/225/Revision 5). Nuclear Security Series No. 27-G, IAEA, Vienna, 2018.

[9] BRASIL - Decreto no 7.722, de 20 de Abril de 2012. Presidência da República do Brasil, Brasília, 2012. Disponível em: <http://www.planalto.gov.br/ccivil_03/_ato2011014/2012/decreto/D7722.htm>. Acessado em: 07/06/2018.

[10]IAEA - International Atomic Energy Agency. Categorization of Radioactive Sources. Safety Standards Series No. RS-G-1.9, AIEA, Vienna, 2005.

[11]CNEN - Comissão Nacional de Energia Nuclear. Licenciamento de Instalações Radiativas, CNEN NN 6.02, CNEN, Brasil, 2017.

[12]CNEN - Comissão Nacional de Energia Nuclear. Requisitos de Segurança e Proteção Radiológica para Serviços de Radiografia Industrial, CNEN NN 6.04, CNEN, Brasil, 2013.

[13]CNEN - Comissão Nacional de Energia Nuclear. Requisitos de Segurança e Proteção Radiológica para Serviços de Radioterapia, CNEN NN 6.10, CNEN, Brasil, 2014.

[14]CNEN - Comissão Nacional de Energia Nuclear. Proteção Física de Unidades Operacionais da Área Nuclear, CNEN NE 2.01, CNEN, Brasil, 2011. 